\title{
Enlightenment of Second Language Acquisition from Language Thinking in English Teaching
}

\author{
Yanmei Zhao \\ Tianhe College, Guangdong Polytechnic Normal University, Guangzhou Guangdong, 510000, \\ China
}

Keywords: English Teaching, Language thinking, Second language acquisition

\begin{abstract}
Actually second language acquisition is a very complex psychological process, language thinking plays a very important role in the process of second language acquisition, therefore, exploring the relationship between language and thought is an essential part of the study of second language acquisition. The relationship between language and thought has always been a controversial topic, the masters in the linguistics field have put forward different views on it, such as Wolf and Sapir tend to believe that language thinking has a direct impact on foreign language learning . This article starts from the perspective of the relationship between language and thinking, study the role of language thinking of second language acquisition and social intercourse in English teaching, and carry on the discussion on how to eliminate the interference of mother tongue thinking and achieve agreement between thought and target language.
\end{abstract}

\section{Introduction}

The language thinking form of learners is a very important factor among the influences of the second language acquisition , whether the second language learners use their mother tongue thinking or use foreign language thinking to learn, which will directly affect the effectiveness of language learning and the quality of language output, therefore, the study of language thinking has opened up a new perspective for the study of language acquisition, and it provides a new learning method for the second language acquisition. Language thinking is getting more and more attention in the second language acquisition process, the article discusses the role of language thinking in the second language acquisition from the aspects of language and thinking, the differences between English and Chinese thinking, and gets the corresponding conclusion.

\section{The relationship between language and thought}

The relationship between language and thought has always been a controversial topic, some linguistic experts study in the view of monism and they consider the purpose and function of language and thinking are basically the same, the different is that language appears on the surface and thinking is internal. view of dualism thinks that language and thinking are two completely different substance, even if human beings cannot speak but the thinking still exist. Generally speaking, experts and scholars believe there are close relations existed between language and thinking, the two aspects cannot be separated. Dwisht Bolinger and Donald Sears these two scholars believe that thinking is produced in some region of the human head [2], the thinking needs to be expressed in the form of language and pass to others. Therefore, the relationship between language and thought is like a part of the human body, language is an essential step for elaboration of thinking, it is a part of thinking and reflect a person's thinking mode in a certain extent.

Sapir and Wolf put forward Sapir and Wolf hypothesis about the relationship between language and thought, they think that the language form determines the user's thinking, what kind of world language describe to us we will see what kind of world. There are a variety of languages in the world, therefore, people who use different languages have different view of the world. The propose of Sapir and Wolf hypothesis opens the door of language and thinking for us. Fordor Jerry once put forward the hypothesis of language and thinking which is called "thought language hypothesis" , this 
hypothesis considers that thinking is generated in the mental language, if human brain is a computer, thinking is the sign existing in mind, that is to say, if there is no thinking language there will be no thinking and language. Although this hypothesis has caused some controversy when it is put forward, but it provides a new perspective for us to probe into language and thinking in certain extent.. If thinking language is existing, then there are two kinds of thinking in our minds, that is language thinking and non language thinking.

Chomsky has once proposed the theory of language generation. he believes that the brain calculation system is similar to the language generating system, both have the characteristics of voice, semantics, structure. Language faculties can generate an endless, personalized language, which is the utterance of human, these words reflect a person's thinking characteristics and it is the external performance of a person's mental language . Chomsky's explanation can be determined that thinking is the essential attribute of language, recognize the thinking characteristics of language, we need to start with the uniqueness of language thinking and have a thorough research , and not just starts from the relationship between language and thinking or only research language or thinking .

Most linguists at home and abroad have recognized the existence of some kind of thinking that cannot be separated from the language, which is language thinking, at the same time, they recognize the existence of non - language thinking. But some experts and scholars put forward that language and thinking are not synchronized. there is a cross status existing between them, that is to say, these two curves will be a linear development in certain situation or even to coincide, But they separate from each in the end, this also acknowledges that there is a language thinking existing in the human brain, at the same time, there are also non linguistic thinking existing.

\section{The influence factors of second language acquisition}

\section{The main factors that affect second language acquisition.}

\section{Individual factors of learners}

The main process of second language acquisition includes perception, understanding, consolidation and application ,it is very important for the study of the second language acquisition. The main factors that influence the second language acquisition including: learning motivation, learning ability, learning intelligence, etc. besides that, there are some other factors such as age, learning methods and ways of thinking, thus there are a lot of factors that influence the second language acquisition.

This paper divides the factors that affect the two language acquisition into the internal and external factors: internal factors include the transfer of the mother tongue, the way of thinking, the individual difference and the emotional factor; external factors include social factors, language acquisition and communication. The external factor is the objective factor of language learning, internal factor is the most important factor for the acquisition of the second language which includes positive transfer, negative transfer, avoidance and over use.

The influence of mother tongue transfer

German linguists once pointed out that when learner is learning the second language, they will been influenced by the native language system no matter in any conditions. Some scholars put forward that mother tongue will have an impact on learning of the second language whether it is positive or negative.

\section{Learning method}

Most Chinese students learn foreign languages through translation method, In spoken or written language, they will firstly use the mother tongue thinking and then translated it into English for expression. This shows that the influence of mother tongue thinking is existing in any situation.

\section{The role of language thinking in second language acquisition.}

American structural linguistics Edward-Sapir has proposed : "Language is a tool and thinking is a product"[3] He thinks that thinking is a reflection the objective world from the human brain, language is the main tool for people to communicate and exchange ideas, it is the main material shell of thinking, thinking precedes language and creates language, but it also has some dependence on 
language, because language can fix a person's thinking mode, thus, language and thinking are produced and developed in the process of interaction between people and objective environment, although they have their own laws of development but the development of the them cannot be separated from the human society . That's why different nations have different views of the world, In some certain language and culture environment, the objective social environment is bound to affect a person's thinking ability, thus the difference of thinking ability is one of the important reasons for the difference of language., in contrary, language differences will promote the formation of thinking patterns. Therefore, people in different cultures have different ways of thinking, these differences are not only reflected in the characteristics of the language, but also reflected in the using method. For language learning, we should pay attention to the cultural differences between different nations, look for the common characters of the two languages and cultures, and enhance foreign language learning.

\section{The influence of the differences in English and Chinese language}

\section{Word difference.}

Chinese character is a kind of ideographic characters, it is one of the few characters that its pronunciation separates from its meaning. Therefore, Chinese language is helpful to the development of the user's thinking ability. English is alphabetic writing, users cannot see its meaning from the glyph, it can only express the idea through the grammar logic relations. Therefore, English can exercise the user's abstract thinking ability.

\section{Language structure difference.}

English belongs to the Indo European language system, compared to Chinese its structure is relatively compact, Chinese belongs to Sino Tibetan language system, its structure is relatively loose. In English, if you want to express a clear concept you need to use the associated words frequently, while Chinese have no fixed word order, users only need to clearly express the point of view.

\section{Word order difference.}

English pays attention to form, rigorously structured, the compound sentence is usually set the predicate verb as the center, and use all kinds of related words to connect with other sentences, showing a space chart expand from center to outside; Chinese mainly show as parataxis, the order of compound sentence usually arranged with the development of time or logic, presenting in linear form, which reflects the analysis and logical thinking of the English language and intuitive thinking of Chinese

\section{Voice difference.}

In English, the active voice and the passive voice are equally important, but Chinese usually take the active voice as maim body, even passive sentences are used to express in the active form. This different form of expression of the English and Chinese also causes different thinking.

\section{Discourse differences.}

The English discourse structure is in linear, the paragraph starts from a topic sentence, the topic is given out directly at the beginning of the article, each paragraph also started in the form of a topic sentence, which belongs to typical deduction and induction form, the Chinese discourse structure is showing as spiral type, the theme of the text is generally not directly explained, but firstly makes various forms of elaboration and then makes a final summary. The difference of discourse structure shows the linear thinking of English and the spiral thinking of Chinese.

From this we can know that thinking is the reflection to the objective world of the human brain, it also can reflect the influence on thinking from language. Therefore, understanding the differences between English and Chinese and two kinds thinking characteristics are the basis of learning foreign language. British and American people emphasize the reason in thinking and pay attention to analysis, As a language , English is a form language, a grammatical means need to be expressed by the change of form; Chinese focus on the integrity and pay attention to the grasp of ideas, therefore, Chinese grammar is usually expressed in the form of parataxis. 


\section{The formation of English acquisition thinking}

\section{The formation of English thinking.}

English Teaching in our country is not a strange concept now, we have a long history in English learning and teaching in China, but there is a wrong idea for English Teaching in our country for a long time, believing that only through the mother tongue can we establish direct contact with foreign language, therefore, most of the English learning is just the process of translation, until the fifty or sixty's this concept has been put right. In the primary stage English Teaching often depends on the learner's mother tongue, learners usually learn English by the method of translating words and sentences, which leads to the mother tongue thinking relatively active in English learning.

Behavioral psychology considers that the transfer of the mother tongue in the second language acquisition process is indeed existed, we should not completely repel the role of mother tongue in learning. The conclusion of many second language acquisition: generally speaking the effect of mother tongue in second language acquisition process is negative, because learners tend to use their mother tongue thinking for foreign language learning, it interferes with the correct understanding of foreign language in a certain extent. Psychological linguistics believe that: People cannot get rid of mother tongue thinking, but it is not completely unable to overcome. Comparative analysis hypothesis believes that through the comparison between mother tongue and target language, learners can find out the difficult study points and predict the errors in the learning process. The process that learners get rid of the bondage of mother tongue thinking and gradually establish foreign language thinking is the process to master a language. In the process of foreign language thinking formation, the formation of language sense is the process of getting rid of mother tongue thinking, it needs regular exercise and exchange practice to achieve the purpose .

\section{Language thinking and communication.}

How communicators think depend on their language proficiency, only by using their proficiency language can they achieve the purpose of communication . Communication is the process of encoding and decoding process between the two parties[5], when using the same language for communication, because of the unity thinking and language thinking synchronization, therefore, the correct rate of encoding decoding is high and communication between two parties can go smoothly. If two parties communicate in foreign language, encoding and decoding process becomes complex, thought and language separated, the two sides need to translate the sentence into their own language and then understand it, such kind of communication tends to be lagged behind. When they directly think in a foreign language, foreign language is directly linked to thinking, its adjustment ability is much stronger, which means foreign language thinking makes the second language communication more effective.

Research on second language acquisition shows that the direct link between the target language and the thought can be realized. In the second language acquisition process, the production of the intermediate language system is the process of getting rid of the mother tongue thinking, intermediate language is a unique language system which is different from the first language and also different from the target language, its formation means that learners have formed a language knowledge system which is independent from the mother tongue and target language in the process of learning, it means that language learning process is no longer a simple translation, but it has formed a direct link between the target language and thinking in learners' mind.

\section{Conclusion}

The relationship between language and thought has a vital importance in the process of second language acquisition, with the continuous in-depth study of the experts and scholars, we have found that the way of human thinking can be divided into linguistic and non linguistic thinking, the two thinking is not the same in second language acquisition, there is a separation between them in some certain extent. For future English teaching in China, teachers should realize the importance of this problem and pay attention to cultivation of students' foreign language thinking ability, build a direct 
link between English learning and thinking by comparing the similarities and differences between English and Chinese, and help students form a foreign language thinking.

\section{References}

[1] Zhu Lili. The Enlightenment for English teaching from the critical period theory of second language acquisitio. English Square (The last ten-day period of a month) 2013(11):65-66.

[2] Zhu Weili. Discussion on enlightenment of second language acquisition theory for college English Teaching. Intelligence,2015(6):253-253.

[3] Wang Zhuo. Discussion on enlightenment of second language acquisition theory for Higher Vocational College English Teaching. Intelligence, 2014(32):54-55.

[4] Guo Zhen. Enlightenment of second language acquisition theory for the English teaching in China. Overseas English, 2014(4):35-38,49.

[5] Jiang Lu. An analysis of the positive effect of Chinese thinking in English class -- English teaching under the perspective of second language acquisition theory. New generation(Last half month). 2015(1):12-13. 